\title{
OPTIMAL LOWER BOUND FOR THE GAP BETWEEN THE FIRST TWO EIGENVALUES OF ONE-DIMENSIONAL SCHRÖDINGER OPERATORS WITH SYMMETRIC SINGLE-WELL POTENTIALS
}

\author{
MARK S. ASHBAUGH AND RAFAEL BENGURIA
}

(Communicated by George R. Sell)

\begin{abstract}
We prove the optimal lower bound $\lambda_{2}-\lambda_{1} \geq 3 \pi^{2} / d^{2}$ for the difference of the first two eigenvalues of a one-dimensional Schrödinger operator $-d^{2} / d x^{2}+V(x)$ with a symmetric single-well potential on an interval of length $d$ and with Dirichlet boundary conditions. Equality holds if and only if the potential is constant. More generally, we prove the inequality $\lambda_{2}\left[V_{1}\right]-\lambda_{1}\left[V_{1}\right] \geq \lambda_{2}\left[V_{0}\right]-\lambda_{1}\left[V_{0}\right]$ in the case where $V_{1}$ and $V_{0}$ are symmetric and $V_{1}-V_{0}$ is a single-well potential.
\end{abstract}

\section{INTRODUCTION}

General bounds on the gaps between consecutive eigenvalues of Schrödinger operators have been the object of considerable attention recently. The gap $\lambda_{2}-$ $\lambda_{1}$ between the first two eigenvalues is of particular interest because it represents the first excitation energy. Singer, Wong, Yau, and Yau [8] proved the lower bound

$$
\lambda_{2}-\lambda_{1} \geq \pi^{2} / 4 d^{2}
$$

for the eigenvalues of the operator $H=-\Delta+V(x)$ acting on $L^{2}(\Omega)$ with Dirichlet boundary conditions where $\Omega$ is a bounded convex subset of $\mathbf{R}^{n}$ and $V$ is a convex potential; the quantity $d$ is the diameter of $\Omega$. More recently, $\mathrm{Yu}$ and Zhong [9] improved estimate (1) to

$$
\lambda_{2}-\lambda_{1} \geq \pi^{2} / d^{2}
$$

under the same hypotheses.

Received by the editors December 16, 1986.

1980 Mathematics Subject Classification (1985 Revision). Primary 34B25.

Key words and phrases. Schrödinger operators, eigenvalue gaps.

The first author was partially supported by grants from the Research Council of the Graduate School, University of Missouri-Columbia and the Programa de las Naciones Unidas para el Desarrollo (PNUD grant CHI-84-005).

The second author was partially supported by the Departamento de Investigacion y Bibliotecas de la Universidad de Chile (Grant E-2486-8612) and by FONDECYT grant 1238-86. 
In this note we derive the optimal bound

$$
\lambda_{2}-\lambda_{1} \geq 3 \pi^{2} / d^{2}
$$

for the one-dimensional case under the additional assumption that $V$ is a symmetric potential. In fact, we prove (3) under the assumption that $V$ is a symmetric "single-well" potential; by this we mean that $V$ is first decreasing and then increasing on the interval in question (of course, for $V$ symmetric the transition from decreasing to increasing must take place at the midpoint).

In addition to the articles cited above there are several other recent articles which present results which are in a sense complementary to our investigation. Whereas our results are specialized to the single-well case these other results are of most interest in the case of multiple-well potentials. The results in [5-7] provide general bounds on eigenvalue gaps; earlier work of Harrell $[3,4]$ and others investigated the behavior of gaps in the context of perturbation theory.

\section{THE MAIN RESULTS}

We consider the two Schrödinger operators $H_{0}=-d^{2} / d x^{2}+V_{0}(x)$ and $H_{1}=-d^{2} / d x^{2}+V_{1}(x)$, both acting on $L^{2}(0, \pi)$ with Dirichlet boundary conditions and with both $V_{0}$ and $V_{1}$ symmetric with respect to $x=\pi / 2$ and in $L^{1}(0, \pi)$. We work with the interval $[0, \pi]$ here for convenience; our general results will follow trivially by rescaling. Let $\left(\lambda_{1}, u_{1}\right)$ and $\left(\lambda_{2}, u_{2}\right)$ be the first two eigenpairs of $H_{1}$ and let $\left(\mu_{1}, v_{1}\right)$ and $\left(\mu_{2}, v_{2}\right)$ be the corresponding quantities for $H_{0}$ (here an eigenpair denotes an eigenvalue together with its associated eigenfunction).

Definition 1. A potential function $V$ is a single-well potential on the closed interval $I$ if there is a $c \in I$ such that $V$ is nonincreasing for $x \leq c$ and is nondecreasing for $x \geq c$. The value $c$ could be either endpoint of the interval $I$.

Proposition 1. Let $H_{0}$ and $H_{1}$ be as described above. If $V_{1}-V_{0}$ is a single-well potential, then

$$
\lambda_{2}-\lambda_{1} \geq \mu_{2}-\mu_{1}
$$

with equality if and only if $V_{1}-V_{0}$ is constant on $[0, \pi]$.

Proof. We consider the trial function (for $u_{1}$ )

$$
u=\left(v_{1} / v_{2}\right) u_{2}
$$

and use it in the Rayleigh-Ritz inequality $\lambda_{1} \leq\left(u, H_{1} u\right) /(u, u)$. The function $u$ is in the domain of $H_{1}$ since the zeros of $u_{2}$ and $v_{2}$ are at $x=0, \pi / 2$, and $\pi$, are all first-order, and hence all cancel. Henceforth, we shall assume that $v_{1}>0$ on $(0, \pi)$ and that $u_{2}$ and $v_{2}$ are positive on $(0, \pi / 2)$ and negative on $(\pi / 2, \pi)$. These can be accomplished by changes of sign as necessary. 
First we compute

(6)

$$
\begin{aligned}
H_{1} u & =\left(v_{1} / v_{2}\right) H_{1} u_{2}-2 u_{2}^{\prime}\left(v_{1} / v_{2}\right)^{\prime}-u_{2}\left(v_{1} / v_{2}\right)^{\prime \prime} \\
& =\lambda_{2} u-2 u_{2}^{\prime}\left(v_{1} / v_{2}\right)^{\prime}-u_{2}\left[v_{2}^{-1} v_{1}^{\prime \prime}-v_{1} v_{2}^{-2} v_{2}^{\prime \prime}-2 v_{1}^{\prime} v_{2}^{-2} v_{2}^{\prime}+2 v_{1} v_{2}^{-3}\left(v_{2}^{\prime}\right)^{2}\right] \\
& =\lambda_{2} u-\left(\mu_{2}-\mu_{1}\right) u-2 u_{2}^{\prime}\left(v_{1} / v_{2}\right)^{\prime}+2 u_{2} v_{2}^{-1} v_{2}^{\prime}\left(v_{1} / v_{2}\right)^{\prime} \\
& =\lambda_{2} u-\left(\mu_{2}-\mu_{1}\right) u-2 v_{2}\left(v_{1} / v_{2}\right)^{\prime}\left(u_{2} / v_{2}\right)^{\prime} .
\end{aligned}
$$

It follows that

$$
\lambda_{1}(u, u) \leq \lambda_{2}(u, u)-\left(\mu_{2}-\mu_{1}\right)(u, u)-2 \int_{0}^{\pi} u v_{2}\left(v_{1} / v_{2}\right)^{\prime}\left(u_{2} / v_{2}\right)^{\prime} d x
$$

and hence

or

$$
\lambda_{2}-\lambda_{1} \geq \mu_{2}-\mu_{1}+\frac{2}{(u, u)} \int_{0}^{\pi} u v_{2}\left(v_{1} / v_{2}\right)^{\prime}\left(u_{2} / v_{2}\right)^{\prime} d x
$$

$$
\lambda_{2}-\lambda_{1} \geq \mu_{2}-\mu_{1}+\frac{4}{(u, u)} \int_{0}^{\pi / 2} u v_{2}\left(v_{1} / v_{2}\right)^{\prime}\left(u_{2} / v_{2}\right)^{\prime} d x .
$$

The result $\lambda_{2}-\lambda_{1} \geq \mu_{2}-\mu_{1}$ will follow if we can show that the integral above is nonnegative. To show this, we first observe that by our assumptions $u$ and $v_{2}$ are both positive on $(0, \pi / 2)$. Hence it remains to consider $\left(v_{1} / v_{2}\right)^{\prime}$ and $\left(u_{2} / v_{2}\right)^{\prime}$. We compute

$$
\left(v_{1} / v_{2}\right)^{\prime}=\left(v_{2} v_{1}^{\prime}-v_{1} v_{2}^{\prime}\right) / v_{2}^{2}
$$

and

Therefore,

$$
\begin{aligned}
\left(v_{2} v_{1}^{\prime}-v_{1} v_{2}^{\prime}\right)^{\prime} & =v_{2} v_{1}^{\prime \prime}-v_{1} v_{2}^{\prime \prime} \\
& =v_{2}\left(V_{0}-\mu_{1}\right) v_{1}-v_{1}\left(V_{0}-\mu_{2}\right) v_{2} \\
& =\left(\mu_{2}-\mu_{1}\right) v_{1} v_{2}>0 \text { on }(0, \pi / 2)
\end{aligned}
$$

$$
\left(v_{1} / v_{2}\right)^{\prime}=\left(\mu_{2}-\mu_{1}\right) v_{2}^{-2} \int_{0}^{x} v_{1} v_{2} d t>0
$$

and $\left(v_{1} / v_{2}\right)^{\prime}$ is seen to be positive on $(0, \pi / 2)$. Lastly, we consider

$$
\left(u_{2} / v_{2}\right)^{\prime}=\left(v_{2} u_{2}^{\prime}-u_{2} v_{2}^{\prime}\right) / v_{2}^{2}
$$

From

it follows that

$$
\begin{aligned}
\left(v_{2} u_{2}^{\prime}-u_{2} v_{2}^{\prime}\right)^{\prime} & =v_{2} u_{2}^{\prime \prime}-u_{2} v_{2}^{\prime \prime} \\
& =v_{2}\left(V_{1}-\lambda_{2}\right) u_{2}-u_{2}\left(V_{0}-\mu_{2}\right) v_{2} \\
& =\left[\left(V_{1}-V_{0}\right)-\left(\lambda_{2}-\mu_{2}\right)\right] u_{2} v_{2}
\end{aligned}
$$

$$
v_{2} u_{2}^{\prime}-u_{2} v_{2}^{\prime}=\int_{0}^{x}\left[\left(V_{1}-V_{0}\right)-\left(\lambda_{2}-\mu_{2}\right)\right] u_{2} v_{2} d t .
$$

Since the integral appearing here must vanish at $x=\pi / 2$ and since $V_{1}-V_{0}$ 
is nonincreasing on $(0, \pi / 2)$ by assumption we must either have $V_{1}-V_{0}=$ const. $=\lambda_{2}-\mu_{2}$ or there must be a point $x_{0} \in(0, \pi / 2)$ such that $V_{1}-V_{0}-$ $\left(\lambda_{2}-\mu_{2}\right)>0$ on $\left(0, x_{0}\right)$ and $V_{1}-V_{0}-\left(\lambda_{2}-\mu_{2}\right) \leq 0$ on $\left(x_{0}, \pi / 2\right)$. In either case this shows that $\left(u_{2} / v_{2}\right)^{\prime} \geq 0$ on $(0, \pi / 2)$ and the conclusion $\lambda_{2}-\lambda_{1} \geq \mu_{2}-\mu_{1}$ follows. Moreover, $\left(u_{2} / v_{2}\right)^{\prime}>0$ on $(0, \pi / 2)$ unless $V_{1}-V_{0}=$ const. and the case of equality is characterized as stated in the proposition.

Theorem 1. Let $H=-d^{2} / d x^{2}+V(x)$ be an operator on $L^{2}(a, b)$ with Dirichlet boundary conditions and suppose that $V$ is a symmetric single-well potential. Then its first two eigenvalues satisfy

$$
\lambda_{2}-\lambda_{1} \geq 3 \pi^{2} /(b-a)^{2}
$$

with equality if and only if $V=$ const .

Proof. This follows directly from Proposition 1 by taking $V_{0}=0$ and $V_{1}=V$ and observing that then $\mu_{1}=\pi^{2} /(b-a)^{2}$ and $\mu_{2}=4 \pi^{2} /(b-a)^{2}$.

Remarks 1. From Theorem 1 it follows easily that if $V$ is symmetric and convex then $\lambda_{2}-\lambda_{1} \geq 3 \pi^{2} / d^{2}$ wehre $d$ is the length of the interval as stated in our Introduction. Furthermore, we conjecture that the hypothesis that $V$ is symmetric can be removed; i.e. that $V$ convex is sufficient to insure $\lambda_{2}-\lambda_{1} \geq 3 \pi^{2} / d^{2}$. Unfortunately, our method of proof above, which relies quite heavily on the symmetry hypothesis, seems unlikely to extend to this case.

On the other hand, we emphasize that a symmetric single-well potential need not be convex. Thus our theorem applies to a somewhat different class of potentials than do those of Singer, Wong, Yau, and Yau and of Yu and Zhong. The intersection of these classes is exactly the class of symmetric convex potentials. We know, however, that (8) cannot hold for all single-well potentials (consider the perturbation of $V \equiv 0$ by a small well placed near one endpoint of the interval).

2. In the case of general dimension $n$ with $H=-\Delta+V(x)$ acting on $L^{2}(\Omega)$ with Dirichlet boundary conditions where $\Omega$ is a bounded convex subset of $\mathbf{R}^{n}$ and $V$ is convex we conjecture the bounds

$$
\lambda_{2}-\lambda_{1} \geq\left(\lambda_{2}-\lambda_{1}\right) \mid \Omega_{0}=\text { ball of same volume as } \Omega
$$

and

$$
\lambda_{2}-\lambda_{1} \geq\left.\left(\lambda_{2}-\lambda_{1}\right)\right|_{V=0}
$$

or even

$$
\lambda_{2}-\lambda_{1} \geq\left.\left(\lambda_{2}-\lambda_{1}\right)\right|_{\substack{\Omega_{0}=\Omega \\ V_{0}=0}} \geq\left.\left(\lambda_{2}-\lambda_{1}\right)\right|_{\Omega_{0}=\text { ball of volume }|\Omega|} .
$$

3. The choice of $u=v_{1} u_{2} / v_{2}$ as trial function for $u_{1}$ was motivated by the fact that this reduces exactly to $u_{1}$ in the case where $V_{0}=V_{1}$ or, more 
generally, when $V_{1}-V_{0}=$ const . One might also observe that the integral in (7) reduces to 0 (as it must) in this case due to the factor $\left(u_{1} / v_{2}\right)^{\prime}$ in the integrand.

4. For nonnegative symmetric single-well potentials we now have the optimal inequalities

$$
3 \pi^{2} / d^{2} \leq \lambda_{2}-\lambda_{1} \leq 3 \lambda_{1}
$$

(see [1] for the second of these which actually holds whenever $V$ is a nonnegative potential) with equality in both if and only if $V=0$. While the conclusion $\lambda_{1} \geq \pi^{2} / d^{2}$ is well known the true utility of these results is that they provide tight bounds on $\lambda_{2}-\lambda_{1}$ particularly in cases where $V$ is small. However, it might be noted that even for very large potentials (in an $L^{p}$ sense, say) one can find potentials which come arbitrarily near to saturating either of the inequalities above. Indeed, the first inequality is saturated by taking a constant potential and we can come arbitrarily close to saturating the second by taking potentials which are concentrated near the endpoints of the interval $[a, b]$ (such potentials also come arbitrarily close to saturating the first inequality).

5. The investigations presented above also yield "single-barrier" results. In particular, if $V$ is a symmetric single-barrier potential in one dimension and Dirichlet boundary conditions are in effect then it follows that $\lambda_{2}-\lambda_{1} \leq 3 \pi^{2} / d^{2}$. Results of this type follow by reversing the roles of $V_{0}$ and $V_{1}$ in our Proposition 1 .

Note added in proof (7/12/88). In the proof of Proposition 1 one should first restrict consideration to continuous potentials to insure that the various manipulations carried out there are justified. One can then regain the case of $L^{1}$ potentials by using a limiting argument to show that the inequality (4) continues to hold in that more general setting.

In subsequent work we have discovered that the inequalities (9), (10), and (11) conjectured in Remark 2 cannot hold in general. However, in the important special case where $\Omega$ is a ball in $\mathbf{R}^{n}$ and $V$ is spherically symmetric (central), we have shown that the bound (10) does hold. In general, though, the best that might be hoped for if $\Omega$ is convex and $V$ is a convex function on $\Omega$ is the bound $\lambda_{2}-\lambda_{1} \geq 3 \pi^{2} / d^{2}$ where $d$ is the diameter of $\Omega$. Further discussion of these points and the result alluded to above will be contained in our forthcoming paper Lower bounds for gaps. II. The multi-dimensional case with a spherically symmetric single-well potential.

For discrete (difference equation) analogues of the one-dimensional results presented here, see our paper Some Eigenvalue Inequalities for a glass of Jacobi matrices. In addition, several of these results are summarized in our contribution to the volume Maximum Principles and Eigenvalue Problems in Partial Differential Equations, P. W. Schaefer, editor, Longman Press, to appear in 1988. 


\section{REFERENCES}

1. M. S. Ashbaugh and R. Benguria, Best constant for the ratio of the first two eigenvalues of onedimensional Schrödinger operators with positive potentials, Proc. Amer. Math. Soc. 99 (1987), 598-599.

2. E. B. Davies, Structural isomers, double wells, resonances and Dirichlet decoupling, Ann. Physics 157 (1984), 166-182.

3. E. Harrell, Double wells, Comm. Math. Phys. 75 (1980), 239-261.

4. __ On the rate of asymptotic eigenvalue degeneracy, Comm. Math. Phys. 60 (1978), 73-95.

5. W. Kirsch and B. Simon, Universal lower bounds on eigenvalue splittings for one-dimensional Schrödinger operators, Comm. Math. Phys. 97 (1985), 453-460.

6. W. Kirsch and B. Simon, Comparison theorems for the gap of Schrödinger operators, J. Funct. Anal. 75 (1987), 396-410.

7. S. Nakamura, A remark on eigenvalue splittings for one-dimensional double-well Hamiltonians, Lett. Math. Phys. 11 (1986), 337-340.

8. I. M. Singer, B. Wong, S.-T. Yau, and S. S.-T. Yau, An estimate of the gap of the first two eigenvalues in the Schrödinger operator, Ann. Scuola Norm. Sup. Pisa Ser. 412 (1985), 319333.

9. Q. Yu and J.-Q. Zhong, Lower bounds on the gap between the first and second eigenvalues of the Schrödinger operator, Trans. Amer. Math. Soc. 294 (1986), 341-349.

Department of Mathematics, University of Missouri, Columbia, Missouri 65211

Departamento de Fisica, Facultad de Ciencias Fisicas y Matematicas, Universidad de Chile, Casilla 487/3, Santiago, Chile 